\title{
Materias Primas Liticas y Manufactura de Instrumentos en Tres Localidades de la Costa Oeste del Golfo San Matías (Provincia de Río Negro, Argentina)
}

\author{
Lithic Raw Materials and Artifact Manufacture at Three \\ Archaeological Localities in the Western Coast of San Matias Gulf \\ (Rio Negro Province, Argentina)
}

Jimena Alberti ${ }^{\mathrm{I}}$

\begin{abstract}
RESUMEN
La costa oeste del golfo San Matías (provincia de Rio Negro, Argentina) presenta caracteristicas particulares que la hacen ambiental y geológicamente diferente a la costa norte del mencionado golfo. Nuestras investigaciones tienen como objetivo explorar la dinámica de ocupación durante el Holoceno medio y tardio y evaluar qué papel jugó este espacio en relación con el sistema de movilidad y subsistencia de las poblaciones humanas que habitaron la región, incluyendo la explotación de recursos líticos. En este trabajo se presentan los resultados del análisis del conjunto de instrumentos de tres localidades del área con el objetivo de comenzar a delinear las tendencias en la explotación de materias primas liticas. A partir del uso de indices de reducción observamos diferencias respecto a lo que sucede con los conjuntos liticos de la costa norte del golfo, principalmente en el aprovechamiento de las rocas de grano fino oscuras, abundantes en los conjuntos arqueológicos de ésta y casi ausentes en aquella. No se han detectado tendencias claras en lo que respecta a la intensidad de reducción de los distintos tipos de rocas. Este hecho implica un llamado de atención metodológico respecto de la utilidad del uso de diferentes indices de forma conjunta para el estudio de conjuntos liticos en otras áreas de investigación, ya que no siempre las tendencias detectadas a partir del uso de algunos de ellos son coherentes con las detectadas a partir del uso de otros de forma complementaria.
\end{abstract}

Palabras clave: Conjuntos Liticos, Materias Primas, Manufactura de Instrumentos, Patagonia Argentina; Holoceno Medio-Tardio.

i Instituto Multidisciplinario de Historia y Ciencias Humanas (IMHICIHU) (CONICET). Saavedra 15 5to. piso. Buenos Aires (C1083ACA). Correo-e: jimealberti@gmail.com

Recibido: 28 de junio 2013 Revisado: 24 de octubre 2013 Aceptado: 11 de noviembre 2013 


\begin{abstract}
The western coast of San Matias Gulf (Rio Negro province, Argentina) has certain characteristics that make it environmentally and geologically different from the northern coast of the same Gulf. The archaeological project that has been initiated in the area aims to explore the dynamics of the human occupation in the area during Middle and Late Holocene, and to assess what role it played in relation to mobility and subsistence system of human populations that inhabited the region, including the study of the exploitation of lithic raw materials. This paper presents the results of the analysis of the tool assemblages from three archaeological localities on the West coast of the Gulf, in order to delineate the trends regarding the exploitation of lithic raw materials in the area. Index of reduction proposed by different authors are used together in order to detect general trends. The results show differences with respect to the lithic assemblages of the northern coast of the Gulf, mainly related to the use of dark fine-grained rocks, abundant in the North and almost absent in the West. Finally, no clear patterns were detected in regard to the intensity of reduction of different types of rocks, which is a wake-up call in regard to the usefulness of the use of different reduction index all together.
\end{abstract}

Key words: Lithics, Raw Materials, Artifact Manufacture, Argentinian Patagonia; Middle And Late Holocene.

\title{
INTRODUCCIÓN
}

La manufactura de instrumentos líticos está generalmente condicionada por diversos factores: disponibilidad de materias primas, conocimientos acerca de las actividades de talla o la necesidad de contar con herramientas para la explotación de determinados recursos, entre otros (ver por ejemplo Shelley 1993, Andrefsky 2005, Shiner et al. 2005). En este trabajo presentamos el análisis de los conjuntos instrumentales provenientes de muestreos realizados en loci de tres localidades arqueológicas de la costa oeste del golfo San Matías (provincia de Río Negro, Argentina): Punta Pórfido, Punta Odriozola y Arroyo Verde. Las investigaciones en el área están recién comenzando, por lo que este trabajo presenta los resultados obtenidos a partir del estudio de los conjuntos de estas tres localidades como una primera aproximación al panorama regional. Así, esperamos detectar tendencias preliminares en la explotación de las materias primas líticas y realizar una primera comparación con lo que sucede en la costa norte del golfo, área que ha sido más intensivamente estudiada en el proyecto en curso. Además se combina el uso de distintos índices de reducción propuestos por 
diferentes autores para comprobar si los resultados arrojados por unos son acordes a lo propuesto por otros. De esta forma, se busca aportar información acerca del uso de estos índices que puede ser útil para autores que trabajen en el estudio de la tecnología lítica en otras áreas del mundo. En este sentido, cabe destacar que hemos podido comprobar que no siempre el uso de estos índices de forma complementaria dan resultados similares, tal como sería de esperar. Esto representa un recaudo metodológico que es necesario tomar al realizar análisis usando este tipo de herramientas.

\section{LA COSTA OESTE DEL GOLFO SAN MATÍAS}

La costa oeste del golfo San Matías, que corre de norte a sur y se

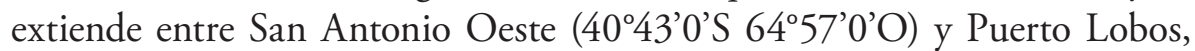
en el límite con la provincia de Chubut ( $42^{\circ} 0^{\prime} 01^{\prime} S 65^{\circ} 04^{\prime} 13^{\prime} \mathrm{O}$ ) (Figura 1). Presenta marcadas diferencias geológicas y estructurales respecto de la costa norte de dicho golfo (para más detalles acerca de estas diferencias, ver Favier Dubois et al. 2008; Favier Dubois y Borella 2011, entre otros). El rasgo geológico principal de este sector es la presencia de la meseta de Somuncurá, una planicie estructural lávica que desciende hacia el mar en forma de pedimentos de flanco (González Díaz y Malagnino 1984). Además de este rasgo particular, la costa puede ser dividida en dos sectores de acuerdo con sus características diferenciales. Al norte de Punta Sierra el ambiente es de tipo sedimentario, con un acceso al mar casi ininterrumpido con restingas de rocas sedimentarias (Favier Dubois y Borella 2011). En cambio, al sur de este punto predomina un ambiente volcánico, interrumpido en algunos sectores por estuarios con playas arenosas y dunas (por ejemplo, los estuarios de los arroyos Salado y Verde) (Favier Dubois et al. 2008, Favier Dubois y Borella 2011). Esta costa es, en general, ambientalmente más homogénea que la parte norte del golfo, con lo que presenta una diversidad de especies marinas menor (Orensanz et al. 1973). 

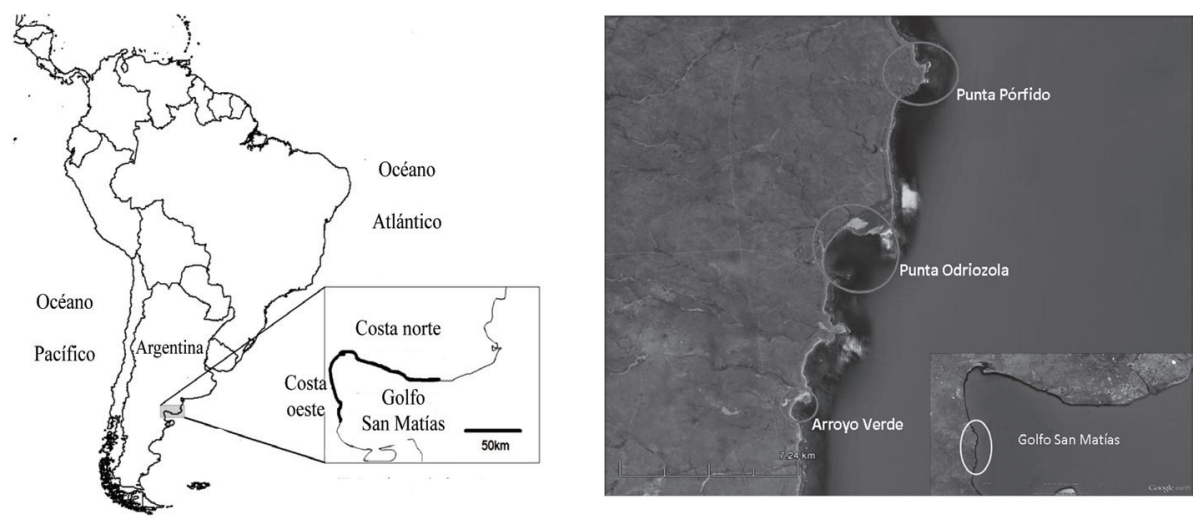

Figura 1. Costa oeste del golfo San Matías ubicada en Sudámerica. Se encuentran señaladas las localidades donde se realizaron los muestreos en la porción sur de esta costa.

Figure 1:Western coast of San Matias Gulf, located in South America. The localities where the samples were taken in the southern portion of the coast are shown.

El estudio de la disponibilidad de materias primas líticas, en proceso, cuenta con datos extraídos de las cartas geológicas y de trabajos de campo de los miembros del equipo de investigación. En esta zona existen fuentes primarias y secundarias de rocas. Las primeras están constituidas por afloramientos porifídicos con vetas y bloques de sílice de diferentes calidades (Cardillo y Scartascini 2007). Además, en trabajos de campo realizados recientemente, hemos identificado una fuente primaria de jaspe asociada a otra de riolita, cuyas calidades para la talla (sensu Aragón y Franco 1997) varían de mala a excelente dentro de la misma fuente (Alberti y Cardillo 2013).

Respecto de las segundas, las fuentes secundarias, cabe destacar que en lugares puntuales del espacio, como por ejemplo en las cercanías del arroyo Verde, han sido localizados nódulos de calcedonia de calidad excelente, que también han sido detectados en depósitos primarios (Cardillo y Scartascini 2007). Además, en algunos sectores de los acantilados han sido identificados depósitos de la Formación Tehuelche, compuestos por rodados de vulcanitas, plutonitas, cuarcitas y sedimentitas (Martínez et al. 2001). Además de estas variedades de rocas, Sánchez (1973) ha propuesto que los tipos de rocas presentes en la costa oeste del golfo San Matías están integrados por areniscas, limonitas, pelitas, pórfidos, granitos, filitas y pizarras.

Finalmente, cabe mencionar la presencia de obsidiana en los loci arqueológicos de esta porción de la costa del golfo que provendrían de distancias que se ubican entre los 225 y los $560 \mathrm{~km}$ lineales desde el área mencionada (Favier Dubois et al. 2009). 
Respecto al material arqueológico, el registro en esta zona es visible debido a la baja tasa de sedimentación sobre las terrazas marinas (Manzi et al. 2009). Hasta el momento han sido localizados 42 loci, más abundantes en el tramo septentrional, donde se registran dunas, cordones litorales y niveles aterrazados (Favier Dubois et al. 2008, Favier Dubois y Borella 2011). Las ocupaciones que han sido datadas se ubican entre los 3200 y los 700 ańos $^{14}$ C AP, aproximadamente (Favier Dubois y Borella 2011, Borella et al. 2013).

Teniendo en cuenta esta información ambiental, sumada a la de tipo arqueológico que ya se tiene del área, se han delineado tendencias respecto del aprovechamiento de la costa oeste del golfo San Matías. La menor disponibilidad de recursos con respecto a la costa norte, la escasa presencia de agua dulce debido al poco desarrollo de aguadas asociadas a depósitos eólicos y la escasez de reparos topográficos, ha dado lugar a la propuesta de que esta zona habría sido utilizada de forma menos intensiva o no redundante, destacándose la presencia de ciertos enclaves atractivos para la ocupación humana (Borella et al. 2007, Favier Dubois y Borella 2011). Esta hipótesis es congruente con lo observado en la diversidad y composición de los conjuntos líticos (Cardillo 2009), y se refleja en la baja presencia de concheros, de evidencia bioarqueológica y arqueofaunística, y de loci utilizados de forma repetida en el tiempo. Todo esto, sumado a la presencia de obsidiana proveniente de lugares distantes (Favier Dubois et al. 2009), ha llevado a pensar que este espacio podría haber funcionado en momentos tardíos como un espacio de circulación, hipótesis que está siendo evaluada en los proyectos de investigación en curso (Favier Dubois y Borella 2011).

\section{La tecnología lítica en la costa oeste del golfo San Matias}

En trabajos previos (Cardillo y Scartascini 2011) se han delineado tendencias en lo que hace a la tecnología lítica de la costa oeste del golfo San Matías. En primer lugar, se ha detectado el predominio de artefactos producidos por actividades de manufactura de instrumentos, como ser lascas internas, externas y desechos no diferenciados. En orden de importancia siguen los instrumentos, siendo los de formatización sumaria y los filos naturales con rastros complementarios los más representados en el registro arqueológico (Cardillo y Scartascini 2011). Además, predominan los filos simples, seguidos de los compuestos y, en menor medida, los dobles. Respecto de los núcleos, en el sector oeste son mayoría los de tipo poliédrico, seguidos por los de lascados aislados (Cardillo y Scartascini 2011). Estos núcleos en general se encuentran descartados con una intensidad de explotación menor que en el sector norte. Cabe destacar que los núcleos de tipo piramidal sólo 
se encuentran presentes en este sector, lo cual estaría relacionado, al menos en parte, con los tipos de formas base empleados para la manufactura de artefactos (Cardillo y Scartascini 2011).

Respecto de las materias primas, se utilizaron preferentemente calcedonias, ópalos y diferentes variedades de sílices, de calidad buena y muy buena para la talla (Cardillo y Scartascini 2007). El sector oeste se destaca en particular por poseer nódulos tabulares de calcedonia de calidad muy buena y excelente, disponibles en sectores puntuales del espacio. En esta área, además, la proporción de rocas de grano fino oscuras (sensu Charlin 2005) (en adelante, $\mathrm{RGFO}^{1}$ ) en los conjuntos arqueológicos es mucho menor que en el sector norte, pero, en cambio, se observan mayores proporciones de rocas síliceas (Cardillo y Scartascini 2011).

En base a la evidencia mencionada, para la costa oeste del golfo San Matías se ha propuesto una mayor inversión de energía en la confección de ciertas clases artefactuales, como los denticulados, lo cual parecería sugerir que constituyen un diseño flexible, a diferencia de los raspadores que presentan una alta estandarización y que habrían sido más conservados (Cardillo y Scartascini 2011). Además, llama la atención la escasa presencia de instrumentos de filos largos, como las raederas, fenómeno que se repite en toda Patagonia septentrional (M. Cardillo com. pers. 2013). Por otro lado, en este sector de la costa el descarte de instrumentos fragmentados habría sido menor que en la costa norte, la abundancia de desechos de talla es también menor y los núcleos están menos intensamente explotados. Todo esto sostiene la hipótesis de un uso menos intenso del sector oeste en comparación con lo que sucede en el norte del golfo San Matías (Cardillo y Scartascini 2011).

\section{Procedencia y CARACTERÍSTICAS DE LA MUESTRA}

ARTEFACTUAL Y METODOLOGÍA DE ANÁLISIS

La muestra analizada en este trabajo está compuesta por un total de 601 artefactos entre los cuales se cuentan desechos enteros y fracturados con talón (los desechos fracturados sin talón han sido dejados fuera para evitar generar sobre-representaciones), instrumentos y núcleos (ver Figuras 2, 3 y 4 para algunos ejemplos). Esta muestra fue recuperada en loci de Arroyo Verde, Punta Odriozola y Punta Pórfido (ver Figura 1). 

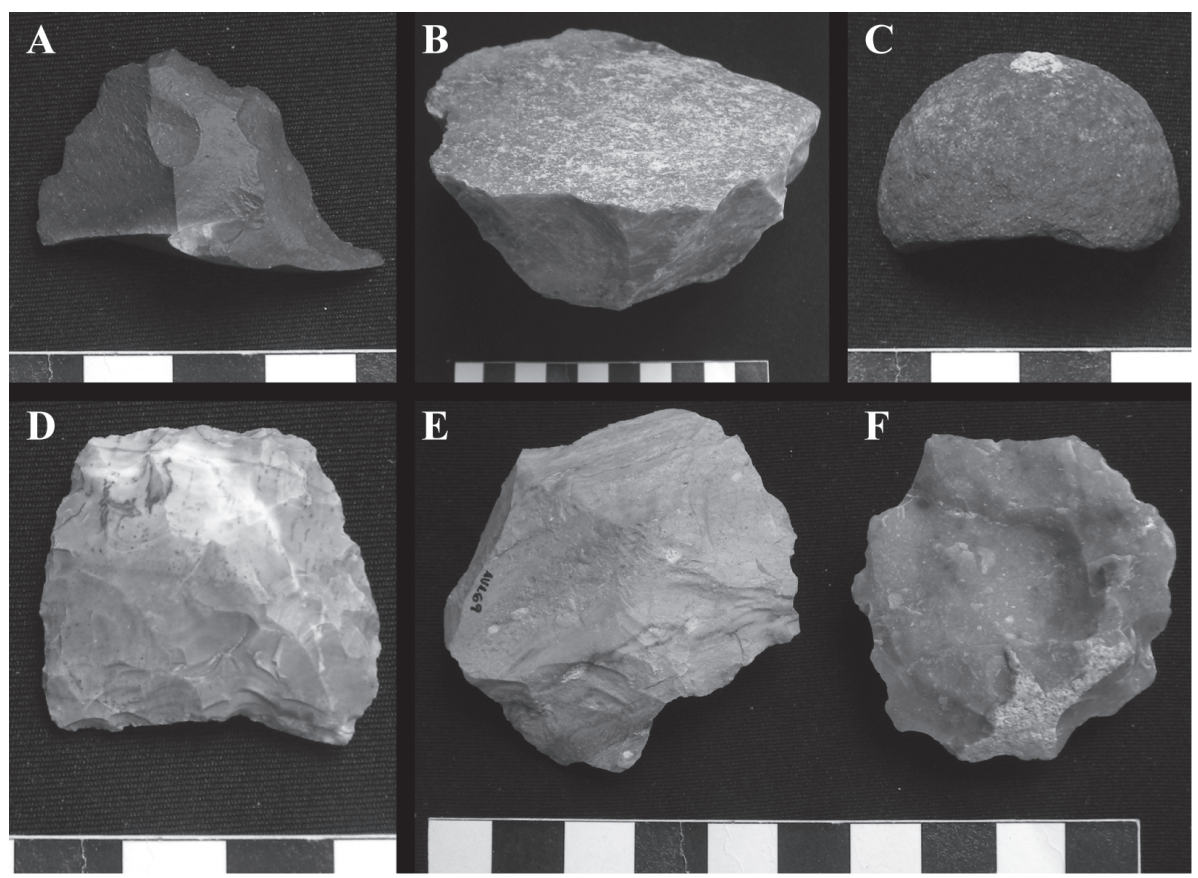

Figura 2: Núcleos e instrumentos recuperados en Arroyo Verde. A) Denticulado (riolita). B) Núcleo (roca silicea). C) Fragmento de bola (roca indeterminada, posiblemente plutónica con alto contenido metálico). D) Bifaz (roca silicea). Ey F) Núcleos (rocas siliceas).

Figure 2: Cores and instruments recovered in Arroyo Verde locality. A) Denticulate (rhyolite). B) Core (siliceous rock). C) "Bola stone" (unidentified rock, possible plutonite with high metal content). D) Biface (siliceous rock). E and F) Cores (siliceous rocks).

La metodología aplicada para la recuperación de los conjuntos fue de tipo distribucional (Franco y Borrero 1999) debido a que el registro se distribuye, a excepción de los concheros, en superficie. Las cuadrículas de recolección, de tamaños variables dependiendo de la densidad de artefactos, se plantearon sobre los sectores con mayores concentraciones artefactuales en cada localidad. Fueron incluidos también en la muestra los hallazgos aislados, ya que se considera que constituyen categorías artefactuales particulares que nos interesa rescatar debido a que, al estar en superficie, estos materiales suelen ser objeto de las actividades de huaqueo de coleccionistas. En algunos casos las cuadrículas de recolección fueron situadas en sectores aledaños a los concheros que fueron excavados, mientras que en otros constituyeron la única evidencia recolectada en los loci.

Para los análisis de orden tecno-morfológico de la muestra artefactual se siguieron los criterios establecidos en Aschero (1975, rev. 1983) y los delineados 
en Hocsman y Escola (2006-2007). Estos últimos autores establecen criterios para estudiar la inversión de trabajo puesta en la manufactura de las diferentes clases artefactuales de un conjunto determinado. Para ello, han utilizado la noción de "clase técnica" a fin de cuantificar la superposición de los lascados que pueden cubrir total o parcialmente la superficie de una o de las dos caras, entrecruzándose o no en el eje medio del instrumento (Aschero y Hocsman 2004). Así, se han establecido ocho clases técnicas en función de una inversión de trabajo decreciente: artefacto con adelgazamiento bifacial, artefacto con reducción bifacial, artefacto con adelgazamiento unifacial, artefacto con reducción unifacial, artefacto con trabajo no invasivo bifacial, artefacto con trabajo no invasivo unifacial, artefacto con trabajo no invasivo alternante y artefacto con trabajo bipolar (Aschero y Hocsman 2004, Hocsman 2006, Hocsman y Escola 2006-2007:76-78). Estas consideraciones van de la mano con la noción de "diseńo utilitario" delineada por Escola (2004), que implica que este tipo de diseño está condicionado, entre otras cosas, por la demanda funcional de cada instrumento, que incluye formas de filos simples que servirían para realizar diversas actividades y también ciertas configuraciones de borde para funciones específicas. Es necesario recordar que esta variable de diseńo adquiriría significado en situaciones desprovistas de stress temporal, con lo que las tareas de manufactura, uso y descarte se darían en el contexto de uso y las actividades de mantenimiento y reparación serían poco frecuentes (Escola 2004). Teniendo esto en cuenta, Hocsman y Escola (2006-2007) proponen cuatro categorías analíticas para abordar los diferentes diseños utilitarios: clase técnica, requerimientos de extracción de la forma base, requerimientos de formatización de la forma base y requerimientos de imposición de forma (para más detalle ver Hoscman y Escola 2006-2007:85-86). Estas categorías han sido tomadas en los análisis junto con la noción de clase técnica para determinar inversión de trabajo en los conjuntos estudiados. 


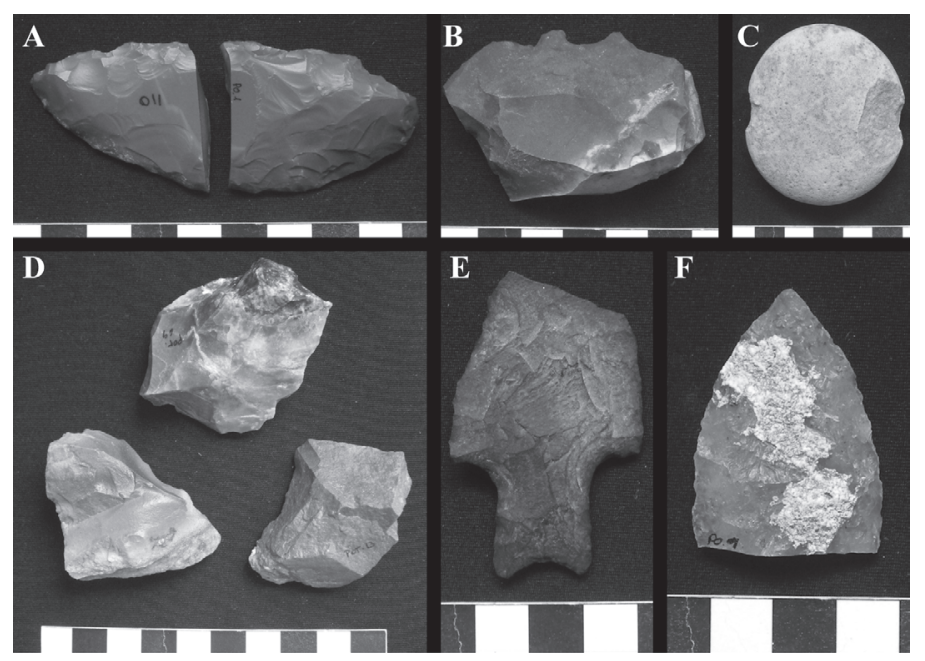

Figura 3: Instrumentos y núcleos recuperados en los muestreos en Punta Odriozola. A) Bifaz (jaspe). B) Denticulado (riolita). C) Pesa lítica (roca sedimentaria). D) Núcleos (rocas siliceas). Ey F) Puntas de proyectil (roca de grano fino oscura y calcedonia).

Figure 3: Cores and instruments recovered in Punta Odriozola locality. A) Biface (jasper). B) Denticulate (rhyolite). C) Stone weight (sedimentary rock). D) Cores (siliceous rocks). E and F) Projectile points (dark fine grained rock and chalcedony).

Con respecto a las intensidades de reducción de los nódulos y el aprovechamiento de las materias primas a nivel general, en trabajos anteriores (Alberti 2013) hemos detectado que en el caso de los conjuntos provenientes de la costa norte del golfo San Matías, no todos los indicadores de reducción propuestos por Dibble (1995), Symons (2003) y Shiner et al. (2005) muestran tendencias similares cuando son aplicados juntos sobre un conjunto en particular. En este sentido, se ha podido comprobar que aquellos indicadores que se comportan de forma similar al ser usados en conjunto (es decir, cuando un índice indica alta o baja reducción para el conjunto de rocas, el o los otro/s indica/n lo mismo) son la razón lascas:núcleos con la mediana de extracciones, los talones facetados y estallados (que indican preparación de la plataforma para buscar cierto producto previamente determinado, y lascados y reducciones intensivas previas a la remoción de una lasca particular) (Symons 2003) y la razón lascas:instrumentos (Alberti 2013). Por otro lado, el uso de la razón lascas con corteza:lascas sin corteza para indicar intensidad de reducción de nódulos arroja resultados similares al ser usada junto con los talones facetados y estallados y con la razón lascas:instrumentos (Alberti 2013). Finalmente, la cuantificación de las proporciones de talones facetados y estallados se comporta de forma parecida a la razón lascas:instrumentos (Alberti 2013). 
Además, hemos podido determinar que hay indicadores de reducción que, al ser usados en conjunto, se comportan como opuestos: mientras algunos indican valores de reducción altos, otros indican valores bajos. Tal es el caso de la razón lascas:núcleos al ser usada con la razón núcleos con corteza:núcleos sin corteza; y el de la razón lascas con corteza:lascas sin corteza al ser usada con la razón núcleos con corteza:núcleos sin corteza o con la mediana de extracciones, entre otros (ver Alberti 2013 para mayor detalle). En este sentido, nos interesó en particular ver qué sucedía con estos indicadores en estos conjuntos de la costa oeste, ya que son, en una primera aproximación, diferentes a los de la costa norte en el sentido de tipos de materias primas presentes, proporción de instrumentos y, además, en lo que hace a la geología del área donde las localidades se ubican.

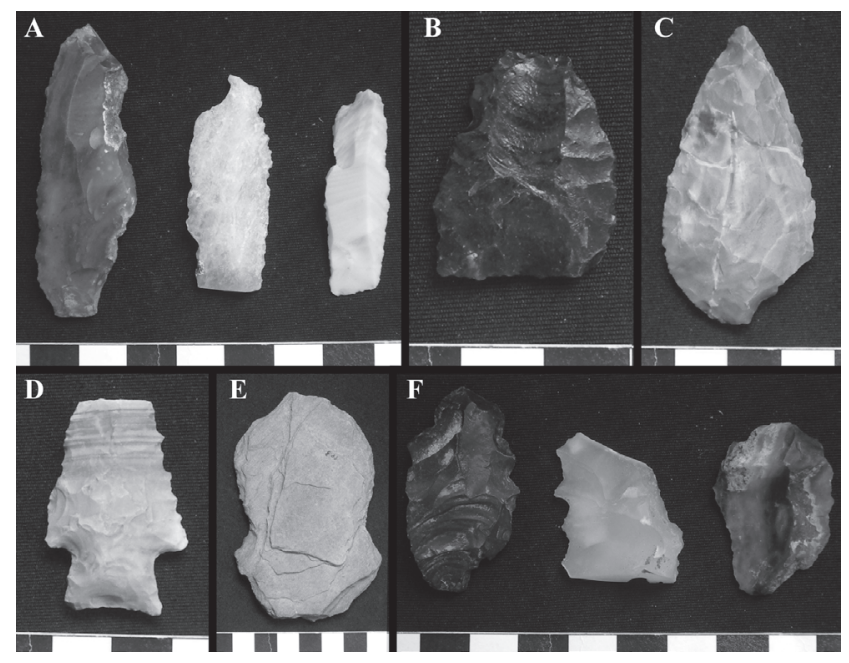

Figura 4: Instrumentos recuperados en Punta Pórfido. A) Filos largos (los dos primeros de calcedonia y el tercero de una roca silicea). B) Punta de proyectil retomada mediante talla bipolar (obsidiana). Cy D) Puntas de proyectil (rocas siliceas). E) Posible azada o hacha (pizarralfilita). F) Denticulados (el primero-manufacturado sobre un núcleo bipolar-y el tercero sobre rocas siliceas, el segundo de calcedonia).

Figure 4: Cores and instruments from Punta Pórfido locality. A) Sidescrapers (the first two made of chalcedony and the third one of siliceous rock). B) Projectile points (obsidian). C and D) Projectile points (siliceous rocks). E) Axe (metamorphic rock). F)

Denticulates (siliceous rocks and chalcedony).

Finalmente, se realizaron algunos test estadísticos para discernir tendencias en la muestra. El primero de ellos fue el análisis de correspondencia para determinar asociaciones entre variables. El segundo implicó la confección de un gráfico de asociación para determinar qué frecuencias observadas 
son mayores que las esperadas por azar. Finalmente, se realizó un test de Kappa (Cohen 1960) para determinar qué indicadores funcionaban bien en conjunto para indicar mayor o menor reducción de los diferentes tipos de rocas. Este coeficiente mide el grado de interrelación entre mediciones y varía entre 0 (ausencia de acuerdo) a 1 (acuerdo perfecto). Los valores cercanos a 0 indican que no existe concordancia entre pares de índices de reducción, y cuanto más cercanos a 1 , más correlación existe. Para otorgarles puntaje a los índices, se les asignó 1 a aquellos que indicaban alta reducción y 0 a aquellos que indicaban baja, para cada tipo de roca analizada. Se espera que si hay concordancia entre los resultados de cada índice, se obtengan puntajes similares por materia prima.

Todas las medidas fueron tomadas mediante el uso de un calibre digital y los datos volcados en planillas de Microsoft Excel confeccionadas para tal efecto. Además se tomaron fotografías de todos los instrumentos y núcleos. Los análisis estadísticos fueron realizados con el software Past 2.1 (Hammer et al. 2001) y el programa R 2.13.0 (R Development Core Team 2005).

\section{Resultados}

La muestra analizada comprende un total de 601 artefactos (Tabla 1), entre los que se recuperaron lascas, núcleos e instrumentos de diferentes materias primas:

\begin{tabular}{|l|c|c|c|c|c|}
\hline & \multirow{2}{*}{ Lascas } & \multirow{2}{*}{ Núcleos } & \multirow{2}{*}{ Instrumentos } & \multicolumn{2}{|c|}{ TOTAL } \\
\cline { 5 - 6 } & & & & $\mathbf{n}$ & $\mathbf{\%}$ \\
\hline Criptocristalinas $^{2}$ & 114 & 24 & 32 & 170 & 28.28 \\
\hline Cuarcita & 3 & 0 & 0 & 3 & 0.49 \\
\hline Cuarzo & 0 & 3 & 0 & 3 & 0.49 \\
\hline Metamórficas & 0 & 0 & 4 & 4 & 0.66 \\
\hline Obsidiana & 9 & 0 & 4 & 13 & 2.16 \\
\hline Piroclásticas & 24 & 7 & 9 & 40 & 6.65 \\
\hline RGFO & 4 & 1 & 1 & 6 & 0.99 \\
\hline Sedimentarias & 47 & 8 & 9 & 64 & 10.64 \\
\hline Sílice & 123 & 16 & 19 & 158 & 26.28 \\
\hline Vulcanitas & 101 & 7 & 26 & 134 & 22.29 \\
\hline Xilópalo & 4 & 2 & 0 & 6 & 6.65 \\
\hline TOTAL & $\mathbf{4 2 9}$ & $\mathbf{6 8}$ & $\mathbf{1 0 4}$ & \multicolumn{2}{|c|}{$\mathbf{6 0 1}$} \\
& $71.38 \%$ & $11.31 \%$ & $17.30 \%$ & \multicolumn{2}{c}{$100 \%$} \\
\hline
\end{tabular}

Tabla 1. Frecuencias absolutas y relativas de tipos artefactuales por tipos de materias primas ( $R G F O=$ rocas de grano fino oscuras).

Table 1: Frequencies of artifacts per raw material (RGFO: dark fine grained rocks). 
En orden decreciente de aparición, los grupos tipológicos más frecuentes en la muestra (ver Tabla 2 para un detalle) son los FNRC (filo natural con rastros complementarios), las pesas, las puntas de proyectil, los denticulados y los raspadores (ver Figuras 2, 3 y 4 para algunos ejemplos de estos instrumentos). En la siguiente tabla se incluyen los instrumentos provenientes tanto de muestreos sistemáticos como dirigidos, y se excluyen de la lista la placa grabada y las dos posibles hachas o azadas.

El primer análisis que se realizó fue la caracterización del conjunto a partir de la propuesta de Escola (2004) y Hocsman y Escola (2006-2007). En primer lugar se discriminaron las diferentes clases técnicas dentro del conjunto instrumental, cuyos resultados se presentan en la Tabla 3, incluyendo provenientes tanto de muestreos sistemáticos como dirigidos. Luego, se establecieron las características restantes para abordar la noción de diseño utilitario (Tabla 4). Cabe aclarar que a pesar de que los filos naturales con rastros complementarios (FNRC) no son artefactos formatizados, los consideramos para la evaluación de la inversión de trabajo (no para la clase técnica) porque son evidencia de actividad expeditiva en busca de un filo útil; sin embargo, no implicarían extraer ninguna forma base en particular, ni formatizarla o imponerle forma.

De la Tabla 3 se desprende que el trabajo no invasivo unifacial es la clase técnica predominante en la muestra. En orden de importancia le siguen la reducción bifacial, el trabajo no invasivo bifacial, el adelgazamiento bifacial y el adelgazamiento unifacial. Todo esto apuntaría a un conjunto en el que predomina una manufactura de tipo expeditivo, sin gran inversión de trabajo en la confección de los instrumentos. En la Tabla 4 se muestran las categorías restantes que, junto con la clase técnica, permiten definir los diseños utilitarios. En este sentido, la percusión dirigida, la ausencia de requerimientos de formatización de la forma base y presencia de requerimientos de imposición de forma dominan en la muestra. 


\begin{tabular}{|l|c|c|}
\hline \multicolumn{1}{|c|}{ Grupo tipológico } & n & \% \\
\hline Bifaz & 4 & 3,96 \\
\hline Bola & 6 & 5,94 \\
\hline Chopper & 6 & 5,94 \\
\hline Cortante & 3 & 2,97 \\
\hline Cuchillo & 3 & 2,97 \\
\hline Denticulado & 10 & 9,90 \\
\hline FNRC & 16 & 15,84 \\
\hline Instrumento fragmentado no diferenciado & 1 & 0,99 \\
\hline Molino & 1 & 0,99 \\
\hline Muesca & 5 & 4,95 \\
\hline Percutor & 2 & 1,98 \\
\hline Pesa & 14 & 13,86 \\
\hline Punta burilante & 2 & 1,98 \\
\hline Punta de proyectil & 11 & 10,89 \\
\hline Punta entre muescas & 2 & 1,98 \\
\hline Raedera & 1 & 0,99 \\
\hline Raspador & 10 & 9,90 \\
\hline RBO & 1 & 0,99 \\
\hline Retoque sumario & 1 & 0,99 \\
\hline Rompecráneo & 1 & 0,99 \\
\hline Sobador & 1 & 0,99 \\
\hline & 101 & 100 \\
\hline
\end{tabular}

Tabla 2. Frecuencia absoluta y relativa de instrumentos presentes en la muestra analizada (FNRC = filo natural con rastros complementarios; $R B O=$ retoque en bisel oblicuo).

Table 2: Frequencies of tools in the assemblage (FNRC=macroscopic edge-wear damage; $R B O=$ burin-like tool).

\begin{tabular}{|l|c|c|c|c|c|}
\hline & \multicolumn{5}{|c|}{ Clase técnica } \\
\cline { 2 - 6 } & $\begin{array}{c}\text { Adelgazamien- } \\
\text { to bifacial }\end{array}$ & $\begin{array}{c}\text { Reducción } \\
\text { bifacial }\end{array}$ & $\begin{array}{c}\text { Adelgazamien- } \\
\text { to unifacial }\end{array}$ & $\begin{array}{c}\text { Trabajo no inv. } \\
\text { bifacial }\end{array}$ & $\begin{array}{c}\text { Trabajo no inv. } \\
\text { unifacial }\end{array}$ \\
\hline Criptocristalinas & 1 & 5 & 0 & 3 & 13 \\
\hline Cuarcita & 0 & 0 & 0 & 0 & 0 \\
\hline Cuarzo & 0 & 0 & 0 & 0 & 0 \\
\hline Metamórficas & 0 & 0 & 0 & 0 & 1 \\
\hline Obsidiana & 2 & 1 & 0 & 1 & 0 \\
\hline Piroclásticas & 0 & 0 & 0 & 1 & 5 \\
\hline RGFO & 0 & 1 & 0 & 0 & 0 \\
\hline Sedimentarias & 0 & 0 & 0 & 0 & 0 \\
\hline Sílice & 0 & 3 & 0 & 4 & 8 \\
\hline Vulcanitas & 0 & 0 & 1 & 0 & 5 \\
\hline Xilópalo & 0 & 0 & 0 & 0 & 0 \\
\hline & $\mathbf{3}$ & $\mathbf{1 0}$ & $\mathbf{1}$ & $\mathbf{9}$ & $\mathbf{3 2}$ \\
\hline
\end{tabular}

Tabla 3. Clases técnicas presentes en la muestra analizada, discriminadas por tipos de materias primas.

Table 3: Technical classes in the assemblage, per raw material. 


\begin{tabular}{|l|c|c|c|c|c|c|}
\hline & \multicolumn{2}{|c|}{$\begin{array}{c}\text { Requerimiento extracción } \\
\text { de la forma base }\end{array}$} & $\begin{array}{c}\text { Requerimiento forma- } \\
\text { tización } \\
\text { de la forma base }\end{array}$ & \multicolumn{2}{c|}{$\begin{array}{c}\text { Requerimiento im- } \\
\text { posición } \\
\text { de forma }\end{array}$} \\
\cline { 2 - 7 } & PD & PND & P & A & P & A \\
\hline Criptocristalinas & 23 & 9 & 7 & 25 & 21 & 11 \\
\hline Cuarcita & 0 & 0 & 0 & 0 & 0 & 0 \\
\hline Cuarzo & 0 & 0 & 0 & 0 & 0 & 0 \\
\hline Metamórficas & 0 & 2 & 0 & 2 & 1 & 1 \\
\hline Obsidiana & 4 & 0 & 3 & 1 & 4 & 0 \\
\hline Piroclásticas & 3 & 3 & 0 & 6 & 5 & 1 \\
\hline RGFO & 1 & 0 & 1 & 0 & 1 & 0 \\
\hline Sedimentarias & 0 & 0 & 0 & 0 & 0 & 0 \\
\hline Sílice & 15 & 4 & 7 & 12 & 13 & 6 \\
\hline Vulcanitas & 4 & 2 & 1 & 5 & 4 & 2 \\
\hline Xilópalo & 0 & 0 & 0 & 0 & 0 & 0 \\
\hline & $\mathbf{5 0}$ & $\mathbf{2 0}$ & $\mathbf{1 9}$ & $\mathbf{5 1}$ & $\mathbf{4 9}$ & $\mathbf{2 1}$ \\
\hline
\end{tabular}

Tabla 4: Frecuencia de atributos de los instrumentos utilizados, junto con la clase técnica, para discernir diferentes diseños utilitarios ( $R F G O=$ rocas de grano fino oscuro, $P D=$ percusión dirigida, $P N D=$ percusión no dirigida, $P=$ presente, $A=$ ausente)

Table 4: Frequency of artifact attributes considered -along with technical class- to distinguish among utilitarian designs ( $R G F O=$ dark fine grained rocks, $P D=$ direct percussion, $P N D=$ indirect percussion, $P=$ present, $A=$ absent).

De la Tabla 3 se desprende que el trabajo no invasivo unifacial es la clase técnica predominante en la muestra. En orden de importancia le siguen la reducción bifacial, el trabajo no invasivo bifacial, el adelgazamiento bifacial y el adelgazamiento unifacial. Todo esto apuntaría a un conjunto en el que predomina una manufactura de tipo expeditivo, sin gran inversión de trabajo en la confección de los instrumentos. En la Tabla 4 se muestran las categorías restantes que, junto con la clase técnica, permiten definir los diseños utilitarios. En este sentido, la percusión dirigida, la ausencia de requerimientos de formatización de la forma base y presencia de requerimientos de imposición de forma dominan en la muestra.

Estos datos se suman a los extraídos a partir del conteo de cantidades de filos en los instrumentos. Esto dejó ver que los instrumentos de filo simple dominan la muestra (68\%), seguidos de los instrumentos bifaciales y, por último, los instrumentos que poseen dos o más filos del mismo o diferente grupo tipológico (17 y 15\%, respectivamente).

Los valores resultantes de las razones usadas para determinar intensidad de reducción se presentan en la Tabla 5. Se observa que la razón lascas:núcleos es más alta en las vulcanitas, seguidas de las rocas 
silíceas, las sedimentarias y por último las criptocristalinas. Cuanto mayor es esta razón, más reducidas habrían sido las rocas en cuestión. De esta forma, las rocas más intensamente aprovechadas serían las vulcanitas y el resto seguirían en orden decreciente. En relación con la mediana de extracciones, es el xilópalo y las RGFO las rocas que presentan la mediana más grande. Sin embargo, estos datos deben ser tomados con el debido recaudo metodológico ya que solamente se encontraron dos núcleos de xilópalo y uno de RGFO en la muestra analizada. Dejando estos casos de lado, las rocas que presentan una mayor mediana de extracciones son las silíceas, seguidas de las piroclásticas (tobas y tobas silicificadas), el cuarzo y las criptocristalinas. Las vulcanitas quedan relegadas al sexto lugar, después de las sedimentarias. Esto indicaría que los núcleos de los que fueron extraídas mayor cantidad de lascas son los de rocas silíceas y así sucesivamente, en orden decreciente.

\begin{tabular}{|c|c|c|c|c|c|c|}
\hline & \multirow[t]{2}{*}{$\begin{array}{l}\text { lascas: } \\
\text { núcleos }\end{array}$} & \multirow[t]{2}{*}{$\begin{array}{l}\text { Mediana de } \\
\text { extracciones }\end{array}$} & \multirow{2}{*}{$\begin{array}{c}\text { lascas con } \\
\text { corteza:lascas } \\
\text { sin corteza }\end{array}$} & \multicolumn{2}{|c|}{$\begin{array}{c}\text { Talones facetados y } \\
\text { estallados. }\end{array}$} & \multirow{2}{*}{$\begin{array}{l}\text { lascas: } \\
\text { instrumen- } \\
\text { tos }\end{array}$} \\
\hline & & & & $n$ & $\%$ & \\
\hline Criptocristalinas & 4.75 & 5.5 & 3.75 & 13 & 35.13 & 3.56 \\
\hline Cuarcita & 0 & 0 & 0 & 0 & 0 & 0 \\
\hline Cuarzo & 0 & 6 & 0 & 0 & 0 & 0 \\
\hline Metamórficas & 0 & 0 & 0 & 0 & 0 & 0 \\
\hline Obsidiana & 0 & 0 & 0.29 & 0 & 0 & 2.25 \\
\hline Piroclásticas & 3.42 & 6 & 0.09 & 1 & 2.70 & 2.66 \\
\hline RGFO & 4 & 8 & 1 & 1 & 2.70 & 4 \\
\hline Sedimentarias & 5.87 & 4.5 & 0.31 & 1 & 2.70 & 5.22 \\
\hline Sílice & 7.68 & 7.5 & 0.24 & 9 & 24.32 & 6.47 \\
\hline Vulcanitas & 14.42 & 4 & 0.84 & 12 & 32.43 & 3.88 \\
\hline Xilópalo & 2 & 21 & 1 & 0 & 0 & 0 \\
\hline
\end{tabular}

Tabla 5: Razones y atributos utilizados para determinar intensidad de reducción en los conjuntos liticos analizados.

Table 5: Ratios and attributes used to determine reduction intensity in the assemblages.

A partir de las propuesta mencionadas sobre la razón lascas con corteza:lascas sin corteza, el análisis de la tabla 5 indica que una mayor reducción se da en el caso de las rocas piroclásticas, seguidas de las silíceas, la obsidiana, las sedimentarias, las vulcanitas, el xilópalo y las RGFO y, por último, las menos intensamente reducidas serían las criptocristalinas.

Al considerar la frecuencia de tipos de talones, se observa que los facetados y estallados, asociados a estados avanzados de reducción, predominan entre las rocas criptocristalinas, seguidas de las vulcanitas y las silíceas y, muy por debajo, las piroclásticas, RGFO y sedimentarias. 
Finalmente, la razón lascas:instrumentos, muestra que la obsidiana presenta la razón más baja, asociándose a una mayor transformación de esta materia prima en instrumentos (Shiner 2004, Shiner et al. 2005). Investigaciones previas han propuesto que en el caso de la costa norte, esta roca proviene de lugares distantes hasta $560 \mathrm{~km}$ lineales, con lo cual es esperable encontrar en los sitios solamente instrumentos o núcleos en sus últimas etapas de reducción, y prácticamente ausencia de desechos de talla (Favier Dubois et al. 2009). Sin embargo, para la costa oeste este factor aún no ha sido evaluado. En el caso de estos sitios, la frecuencia de obsidiana es mayor que en la otra porción de la costa del golfo, con lo cual no podemos hacer extensivas las hipótesis que se están manejando para la costa norte, en la cual se han recuperado artefactos con reserva de corteza y nódulos completos en sitios como Saco Viejo (Favier Dubois et al. 2009). Sacando el caso particular de la obsidiana, la razón más pequeña es la de las rocas piroclásticas, seguidas de las criptocristalinas, las vulcanitas, las RGFO, las sedimentarias y las silíceas, lo que indicaría que de estas rocas, pocas formas base han sido convertidas en instrumentos.

Para obtener un primer acercamiento, entonces, a las estrategias de manufactura de instrumentos en los conjuntos de la costa oeste en relación con los tipos de materias primas utilizados, se realizó un análisis de correspondencia. A pesar de que los dos ejes del análisis explican alrededor del $75 \%$ de la asociación entre las variables, no hay una tendencia gráfica clara que permita discernir asociaciones de grupos de variables, tal como sucedía en el caso de los conjuntos analizados para la costa norte (Alberti 2012b). En el futuro, con la ampliación de la muestra estudiada, probablemente puedan establecerse asociaciones más claras que las actuales.

Para poder detectar aquellas variables que podrían arrojar como resultado la aparición de diferencias estadísticamente significativas al realizar algunos tests, se confeccionó un gráfico de asociación. En el caso de las obsidianas y las vulcanitas, las diferencias estadísticamente significativas aparecen para la presencia de adelgazamiento bifacial y unifacial, respectivamente. Dentro de las metamórficas, este hecho se da para el caso de la percusión no dirigida y, en las RGFO, para la reducción bifacial. En el caso de las obsidianas podría estar relacionado con la proveniencia de esta roca y su entrada a los sitios en forma de instrumentos. En el caso de las vulcanitas, podría relacionarse con la relativamente baja calidad para la talla de estas rocas que no habría permitido la manufactura mediante la talla bifacial. El caso de las rocas metamórficas no llama la atención ya que sobre este tipo de rocas no se han manufacturado instrumentos con una 
alta inversión de trabajo, y el caso de las RGFO sería muy similar al de las obsidianas.

Como conclusión de este análisis, podríamos destacar que las diferencias significativas que pudiesen surgir de la realización de un test estarían mayormente influenciadas por estas tendencias. En un futuro, con la ampliación de la muestra se realizarán análisis que esperamos delineen estas tendencias más claramente.

\section{Discusión}

Tal como se desprende de los datos arriba expuestos, la categoría artefactual más representada dentro de los conjuntos analizados es la de los desechos, seguidos de los instrumentos y por último los núcleos (pese a que la incorporación en el análisis de los materiales provenientes de los muestreos dirigidos podría producir un sesgo hacia los instrumentos). Las materias primas más abundantes son las rocas criptocristalinas (28\%), seguidas de las silíceas (26\%) y las vulcanitas (22\%). Esto implica una primera diferencia respecto de los conjuntos de la costa norte, donde las RGFO juegan un papel más importante dentro de los conjuntos, en particular dentro del bloque tardío (1500-450 años ${ }^{14} \mathrm{C} \mathrm{AP}$ ), conformando casi el 34\% del total de los conjuntos analizados (Alberti 2012a, 2013). En cambio, en las muestras analizadas en este trabajo su presencia no alcanza el $1 \%$ del total. Esto podría estar en relación con la disponibilidad de este tipo de rocas en este sector del espacio: hasta el momento no hemos constatado la presencia de las mismas en las fuentes secundarias de materias primas de la costa oeste (Alberti y Cardillo 2013), las cuales son diferentes a las de la costa norte ya que los cordones litorales del oeste, principales proveedores de este tipo de rocas en el norte del golfo, poseen escasa cantidad de rocas aptas para la talla (C. Favier Dubois com. pers. 2013) (Cardillo y Scartascini 2007, Alberti 2012a).

A diferencia de las rocas criptocristalinas, que sí consideramos que fueron transportadas desde el sector oeste del golfo hacia el norte (Alberti 2012a), las RGFO no habrían tenido una circulación amplia en el espacio ya que, de haberla tenido, su presencia sería más fuerte en el sector oeste. Esto podría deberse a que no tuvieron la calidad suficiente que justificara su transporte más allá de la costa norte, aunque quizás también se relacione con diferencias en la movilidad. De esta manera, el uso de materias primas en la costa oeste habría sido, en un principio, local, aprovechando las fuentes tanto primarias como las secundarias que se encuentran disponibles en el 
área y que ofrecen rocas de calidad para la talla buena a excelente (Alberti y Cardillo 2013).

El caso de la obsidiana es también interesante para destacar. En trabajos previos (Favier Dubois et al. 2009, Alberti 2013) se ha constatado que la presencia de esta roca en la costa norte del golfo es, en general, muy escasa (inferior al $1 \%$ de los conjuntos y en forma de desechos solamente), salvo en lugares puntuales como Saco Viejo, y que, tal como ha sido mencionado más arriba, provendría de lugares distantes hasta 560 kilómetros lineales (Favier Dubois et al. 2009). En la costa oeste, en cambio, la presencia de esta roca alcanza porcentajes superiores, ubicándose por encima del $2 \%$ en muestras aun más pequeñas que las analizadas para la costa norte. Además, no sólo se presenta en forma de lascas sino que también han sido hallados instrumentos confeccionados sobre esta materia prima. La cercanía de la meseta de Somuncurá respecto de los loci de la costa oeste, de donde proviene parte de la muestra de obsidiana recuperada en la costa norte (Favier Dubois et al. 2009), podría ser un factor que explicase la mayor abundancia de esta roca en comparación con lo registrado en la otra porción del golfo.

Respecto de las clases instrumentales, mientras que en la porción norte del golfo predominan los denticulados seguidos por los raspadores, en los loci de las tres localidades analizadas en la costa oeste predominan los FNRC, las pesas y las puntas de proyectil. Denticulados y raspadores aparecen en las mismas proporciones pero por debajo de estas categorías. Por otro lado, al considerar las diferentes clases técnicas de los instrumentos, se destaca una baja inversión de trabajo en la manufactura de los instrumentos (trabajo no invasivo unifacial y ausencia de requerimientos de formatización de la forma base). Sin embargo, y a diferencia de lo que sucede en la costa norte, la clase técnica que sigue en orden de importancia en esta área es la reducción bifacial, que en los otros conjuntos quedaba relegada al último lugar (Alberti 2012b). Esto sugiere la posible existencia de estrategias de manufactura de instrumentos diferentes en ambos sectores de la costa. Por otro lado, observaciones preliminares realizadas durante los trabajos de campo, y en coincidencia con lo planteado por Bórmida (1964), han permitido apreciar que la presencia de hojas en esta área es mayor que en la costa norte, posiblemente indicando no sólo estrategias de manufactura diferentes, sino también estrategias de reducción distintas (M. Cardillo com. pers. 2012). En este sentido, y retomando lo dicho anteriormente, cabe destacar que las fuentes secundarias de materias primas, cordones litorales, principales proveedores de nódulos en la costa norte del golfo, están compuestas principalmente por rocas que no son aptas para la talla, 
con lo que estrategias de reducción distintas serían esperables debido a las diferencias litológicas. Esta hipótesis será evaluada en trabajos futuros.

Finalmente, respecto de la reducción de las rocas, se observa que las vulcanitas son las más reducidas si consideramos la cantidad de lascas estimadas por cada núcleo recuperado. Sin embargo, no son estos núcleos los que presentan una mayor mediana en el número de extracciones, sino que este lugar lo ocupan las rocas silíceas, que estarían disponibles localmente. Al considerar la presencia de corteza en las lascas, la medición indica que las rocas más reducidas fueron las piroclásticas, seguidas de las silíceas. Este dato es de destacar ya que las primeras no serían de origen local o, por lo menos, no hemos detectado presencia de una fuente de piroclastitas en la región, a diferencia de lo que sí sucede con las rocas silíceas que, en mayor o menor medida, de acuerdo con su calidad, serían de origen local. Las vulcanitas otra vez quedan relegadas, incluso por detrás de la obsidiana, de proveniencia no local. Cuando los tipos de talones son considerados, las rocas que aparecen como más reducidas cambian nuevamente. El primer lugar lo ocupan las criptocristalinas, el segundo las vulcanitas y las silíceas el tercero. Finalmente, la cantidad de instrumentos en relación con la cantidad de lascas, dejando de lado el caso particular de la obsidiana, sugiere que las rocas piroclásticas son las que más han sido convertidas en instrumentos, seguidas de las criptocristalinas y las vulcanitas en tercer lugar. De acuerdo con esta razón, las rocas silíceas quedan relegadas al final de la lista.

De estos análisis se desprende que no hay un patrón claro de reducción de rocas. Mientras algunas aparecerían como las más reducidas al tener en cuenta ciertas razones, otras lo hacen al considerar otro tipo de conteos, con lo cual no podemos determinar una tendencia clara en los materiales de las tres localidades analizadas. El hecho más sobresaliente del análisis de este registro es la participación casi nula de las RGFO en el conjunto. Estas diferencias en la reducción de las diferentes rocas podrían tener que ver con cuestiones que hagan a las materias primas en sí o a su forma de abastecimiento, lo cual puede redundar en diferentes estrategias de talla e intensidades de explotación (ver un ejemplo en Borrazzo et al. 2010).

Para poder determinar, entonces, cuáles indicadores muestran tendencias similares, se realizó un test de Kappa (ver Alberti 2013). Para el conjunto total de materias primas, las razones lascas:núcleos y lascas:instrumentos, y la mediana de extracciones de los núcleos se comportan de la misma manera usadas en conjunto. No sucede esto con el resto de los indicadores. Por otro lado, la razón lascas con corteza:lascas sin corteza y la cuantificación de talones facetados y estallados funcionan bien juntos, ya 
que el test da un valor de Kappa de 0.57, con un p-valor de 0.12 (aunque el resultado no es significativo, probablemente por el tamaño de la muestra). Sin embargo, este segundo conjunto de indicadores no funcionaría bien utilizándolo junto con el primero, ya que mientras los indicadores del primer grupo señalarían alta reducción, los del segundo indicarían baja y viceversa, excepto en el caso de las rocas criptocristalinas, donde todos los indicadores funcionarían bien en conjunto. El caso de las vulcanitas también se separa parcialmente del resto, ya que mientras que la razón lascas con corteza:lascas sin corteza funcionaría a la inversa de los indicadores del primer grupo, los tipos de talones funcionarían acorde a estos indicadores. Asimismo, es necesario considerar como hipótesis que quizás otros criterios más allá de los meramente tecnológicos puedan haber estado en juego al momento de decidir acerca de la explotación de algún tipo de roca en particular, como es el caso, por ejemplo, de las tobas del Chorrillo Miraflores (Borrazzo et al. 2010).

Así, vemos que mientras que este grupo de indicadores funcionó bien para ver intensidad de reducción en los conjuntos de la costa norte (Alberti 2013), no es el caso de los conjuntos de la costa oeste, ya que vemos el agrupamiento de los indicadores en dos grupos diametralmente opuestos que en este caso no servirían para trabajar en conjunto. Esto puede estar relacionado con el número de casos analizado, con lo que estos tests deberían ser realizados nuevamente luego de ampliar la muestra estudiada en el futuro.

\section{Conclusiones}

Tal como se desprende de los análisis realizados en el marco de este trabajo, existen diferencias importantes respecto de lo que ya se ha estudiado para el caso de los conjuntos líticos de la costa norte. En primer lugar, el hecho más destacable es la casi ausencia de RGFO en los conjuntos de la costa oeste, tipo de roca que es mayoritario en el sector septentrional del golfo, por lo menos para los momentos más tardíos de la ocupación (Alberti 2012a). Tal como ya mencionamos, este tipo de roca no se encuentra en abundancia en las fuentes de materias primas secundarias de la porción del golfo estudiada en este trabajo. Este hecho, sumado a su escasa presencia en el registro, estaría apuntando a una ausencia de transporte de este tipo de roca, pese a que su calidad para la talla es, en general, muy buena. Esta falta de transporte podría deberse a la abundancia relativa de otras rocas de buena calidad para la talla existentes en las fuentes de la costa oeste o, por ejemplo, a que los grupos que ocuparon la costa oeste no frecuentaron la costa norte del golfo San Matías, por lo menos para los momentos en los que estos sitios 
fueron ocupados. Quizás también responda a un sentido de circulación sur-norte y oeste-este cuyo resultado sería la falta de representatividad de materiales de la costa norte en el sector oeste.

Los indicadores de intensidad de reducción para los distintos tipos de rocas no arrojan resultados claros, a pesar de haberse utilizado aquellos que en el caso de la costa norte funcionaron bien en conjunto (Alberti 2013). Esto puede tener que ver con los tamaños de las muestras de la costa oeste que aún son pequeñas o con que no se prefirió ningún grupo de rocas por sobre otro para reducir más o menos intensamente en busca de formas base particulares para la manufactura de los instrumentos. También esto puede estar en relación con la disponibilidad de materias primas líticas debido a que ésta es diferente en la costa oeste respecto de la norte.

En relación con los tipos de instrumentos, la presencia mayoritaria de FNRC y de diseños utilitarios que no habrían requerido gran inversión de trabajo para la manufactura de las herramientas, estaría apoyando la hipótesis de un uso no reiterado o esporádico de este espacio, quizás para el aprovechamiento puntual de algún tipo de recurso como los peces, cuya explotación se encuentra evidenciada en la gran cantidad de pesas líticas que fueron recuperadas en el área (Favier Dubois y Borella 2011, Borella et al. 2013). Otra hipótesis que podríamos plantear es el recambio de instrumental que se habría utilizado en otros espacios, como por ejemplo las puntas de proyectil, que en todos los casos se encontraron fragmentadas. Debido a que las investigaciones en el área se han comenzado a desarrollar en forma intensiva recientemente, el planteo de suposiciones como éstas es aún muy preliminar. Sin embargo, son cuestiones que deben ser tenidas en cuenta al momento de abordar el estudio del registro arqueológico de la porción sur de la costa oeste del golfo San Matías. Además, restan estudiar más profundamente los loci ubicados en la porción norte de la costa oeste que, tal como ya se ha mencionado (ver supra), presenta características geomorfológicas y geológicas diferentes a la parte sur, con una disponibilidad de recursos distinta y, consecuentemente, un registro arqueológico que puede dar cuenta de otro tipo de uso del espacio en este caso.

A partir de la aplicación de diferentes índices de reducción en estas muestras hemos podido observar que no siempre éstos arrojan resultados acordes entre sí cuando son utilizados en conjunto. Mientras que algunos proponen una reducción intensa, otros dan como resultado que son rocas que no fueron muy reducidas. Esto ya ha sucedido, aunque no en todos los casos, al utilizarlos en otras muestras más grandes (ver Alberti 2013), con lo que consideramos que no es un problema de tamaño de muestra, 
sino que tiene que ver con el uso de los índices en sí. Esto llama la atención acerca de los recaudos metodológicos necesarios al utilizar este tipo de estudios, recaudos que deben ser tomados en cuenta al realizar inferencias a partir del análisis de un conjunto en particular. Consideramos que el uso complementario de los índices de reducción y de intensidad de explotación de rocas propuestos por diferentes autores puede ofrecer un panorama más amplio respecto de lo sucedido en relación con la explotación de rocas de un conjunto en particular.

El análisis de los conjuntos líticos de tres localidades que hemos realizado en este trabajo es consistente con la hipótesis de un uso no reiterado o esporádico del espacio, tal como había sido propuesto en trabajos previos (Cardillo y Scartascini 2011, Favier Dubois y Borella 2011). Sin embargo, la presencia de algunos indicadores de aprovechamiento intensivo de ciertos recursos (pesas líticas) llama la atención acerca de lo que sucede en la porción sur de la costa oeste del golfo San Matías. Además, cabe destacar la elevada presencia de artefactos de molienda en distintos estados de agotamiento que se observaron en este sector y no en la parte norte de la costa oeste (M. Cardillo com. pers. 2013). Trabajos de campo y análisis a futuro ayudarán a corroborar o descartar estas hipótesis iniciales.

Agradecimientos: A Cristián Favier Dubois y Marcelo Cardillo por los comentarios sobre el manuscrito de este trabajo. A Florencia Borella y Federico Scartascini por la colaboración en los trabajos de campo. A Maximiliano Bestene por el tratamiento de las imágenes. A los evaluadores anónimos por las sugerencias y comentarios realizados.

\section{Notas}

${ }^{1}$ Mediante el análisis de cortes delgados se ha podido determinar que las RGFO de la costa norte del golfo San Matías son de origen volcánico y no sedimentario. Como esta determinación no ha sido hecha aún para la costa oeste, preferimos mantener la sigla RGFO, que agrupa a ambos tipos de rocas, debido a la imposibilidad de discernir tipos litológicos mediante el análisis a ojo desnudo.

${ }^{2}$ Esta categoría de rocas agrupa a calcedonias, jaspes y ópalos, rocas de grano muy fino, de calidad en general excelente para la talla. Han sido agrupadas aquí debido a sus características similares, a su baja frecuencia de aparición en el registro y para facilitar los análisis estadísticos.

\section{BiBLIOGRAFÍA}

Alberti, J. 2012a. "Fuentes de rocas y uso de materias primas líticas en Bahía Final 6, costa norte del golfo San Matías (Río Negro, Argentina)". Intersecciones en Antropología 13: 237-249.

- 2012b. "Primera exploración de la inversión de trabajo en los conjuntos líticos de la costa norte del golfo San Matías, provincia de Río Negro (Argentina)”. 
La Zaranda de Ideas. Revista de Jóvenes Investigadores en Arqueología 8(2):83100

—_ 2013. "Explotación de materias primas líticas e intensidad de reducción de nódulos en la costa norte del golfo San Matías (Río Negro, Argentina) durante el Holoceno medio y tardío". Comechingonia Virtual. En prensa.

Alberti, J. y M. Cardillo. 2013. "Fuentes primarias y secundarias de materias primas líticas en la costa oeste del golfo San Matías (Río Negro, Argentina). Una primera aproximación a su variabilidad espacial”. Trabajo presentado en el XVIII Congreso Nacional de Arqueología Argentina. INCIHUSA (CONICET) - Universidad Nacional de La Rioja. La Rioja.

Andrefsky, W. 2005. Lithics: Macroscopic Approaches to Analysis. Cambridge University Press, Cambridge.

Aragón, E. y N. Franco. 1997. "Características de rocas para la talla por percusión y propiedades petrográficas”. Anales del Instituto de la Patagonia - Serie Ciencias Humanas 25: 187-199.

Aschero, C. 1975. Ensayo para una clasificación morfológica de artefactos líticos aplicada a estudios tipológicos comparativos. Informe presentado al CONICET. Manuscrito en posesión del autor.

- 1983. Ensayo para una clasificación morfológica de artefactos liticos. Revisión del año 1975. Informe presentado al CONICET. Manuscrito en posesión del autor.

Aschero, C. y S. Hocsman. 2004. "Revisando cuestiones tipológicas en torno a la clasificación de artefactos bifaciales". En Temas de arqueología. Análisis lítico, editado por A. Acosta, D. Loponte y M. Ramos, pp. 7-25. Universidad Nacional de Luján, Luján.

Borella, F.; M. Cardillo, C. Favier Dubois, F. Scartascini, J. Alberti, H. Marani y E. Borges Vaz. 2013. "Las ocupaciones humanas entre Punta Pórfido y Punta Odriozola, costa oeste del golfo San Matías: nuevos hallazgos y perspectivas". Trabajo presentado en el XVIII Congreso Nacional de Arqueologia Argentina. INCIHUSA (CONICET) - Universidad Nacional de La Rioja. La Rioja.

Borella, F.; C. Mariano y C. Favier Dubois. 2007. "Procesos tafonómicos en restos humanos en superficie en la localidad arqueológica de Bajo de la Quinta, Golfo San Matías (Río Negro)”. En Arqueología de FuegoPatagonia. Levantando piedras, desenterrando huesos... y develando arcanos, editado por F. Morello, M. Martinic, A. Prieto y G. Bahamonde, pp: 403410. Ediciones CEQUA, Punta Arenas. 
Bórmida, M. 1964. “Arqueología de la costa norpatagónica”. Trabajos de Prehistoria 16:7-108.

Borrazzo, K.; M. D’Orazio y M. Etchichury. 2010. "Distribucion espacial y uso prehistórico de materias primas líticas del chorrillo Miraflores en el norte de la Isla Grande de Tierra del Fuego". Revista Chilena de Antropología 22(2):77-97.

Cardillo, M. 2009. Variabilidad en la manufactura y diseño de artefactos en el área costera patagónica. Un enfoque integrador. Tesis para optar al grado de Doctor en Arqueología, Facultad de Filosofía y Letras, Universidad de Buenos Aires, Buenos Aires.

Cardillo, M. y F. Scartascini. 2007. “Tendencias observadas en las estrategias de explotación de recursos líticos en el Golfo de San Matías, provincia de Río Negro, Argentina”. En Arqueología de Fuego-Patagonia. Levantando piedras, desenterrando huesos... y develando arcanos, editado por F. Morello, M. Martinic, A. Prieto y G. Bahamonde, pp: 117-127. Ediciones CEQUA, Punta Arenas.

- _ 2011. "Diversidad artefactual y explotación de materias primas en la costa norpatagónica. Golfo San Matías, Río Negro”. En Arqueología de pescadores y marisqueadores en Nordpatagonia. Descifrando un registro de más de 6.000 años, editado por F. Borella y M. Cardillo, pp: 67-86. Dunken, Buenos Aires.

Charlin, J. 2005. "Utilización de materias primas líticas en el campo volcánico Pali Aike (Pcia. de Santa Cruz, Argentina). Una primera aproximación a partir del análisis de núcleos”. Werkén 7:39-55.

Cohen, J. 1960. "A coefficient of agreement for nominal scales". Educational and Psychological Measurement 20: 37-46.

Dibble, H. 1995. "Middle Paleolithic scraper reduction: background, clarification and review of the evidence to date". Journal of Archaeological Method and Theory 2(4):299-368.

Escola, P. 2004. "La expeditividad y el registro arqueológico". Chungara. Revista de Antropología Chilena 36:49-60.

Favier Dubois, C. y F. Borella. 2011. "Contrastes en la costa del golfo: una aproximación al estudio del uso humano del litoral rionegrino en el pasado". En Arqueología de pescadores y marisqueadores en Nordpatagonia. Descifrando un registro de más de 6.000 años, editado por F. Borella y M. Cardillo, pp: 13-42. Dunken, Buenos Aires.

Favier Dubois, C.; F. Borella, L. Manzi, M. Cardillo, S. Lanzellotti, F. Scartascini, M. Carolina y E. Borges Vaz. 2008. "Aproximación regional 
al registro arqueológico de la costa rionegrina”. En Arqueología de la Costa Patagónica. Perspectivas para la conservación, editado por I. Cruz y S. Caracotche, pp: 50-68. Universidad Nacional de la Patagonia Austral, Río Gallegos.

Favier Dubois, C.; C. Stern y M. Cardillo. 2009. "Primera caracterización de los tipos de obsidiana presentes en la costa rionegrina”. En Arqueología de la Patagonia - Una mirada desde el último confín, editado por M. Salemme, F. Santiago, M. Álvarez, E. Piana, M. Vázquez y E. Mansur, pp: 349-359. Editorial Utopías, Ushuaia.

Franco, N. y L. Borrero. 1999. "Metodología de análisis de la estructura regional de recursos líticos". En En los Tres Reinos. Prácticas de recolección en el Cono Sur de América, editado por C. Aschero, A. Korstanje y P. Vuoto, pp. 27-37. Magna Publicaciones, San Miguel de Tucumán.

González Díaz, E. y E. Malagnino. 1984. "Geología y recursos naturales de la provincia de Río Negro. Geomorfología”. Actas del IX Congreso Geológico Argentino, vol. I (13), pp: 347-364. San Carlos de Bariloche.

Hammer, O.; D. Harper y R. Ryan. 2001. "PAST Palaeontological Statistics software package for education and data analysis". Palaeontológica Electrónica 4(1):9.

Hocsman, S. 2006. Producción Litica, Variabilidad y Cambio en Antofagasta de la Sierra -ca. 5500-1500 AP-. Tesis para optar al grado de Doctor en Arqueología, Facultad de Ciencias Naturales y Museo, Universidad Nacional de La Plata.

Hocsman, S. y P. Escola. 2006-2007. "Inversión de trabajo y diseño en contextos líticos agro-pastoriles (Antofagasta de la Sierra, Catamarca)". Cuadernos del Instituto Nacional de Antropología y Pensamiento Latinoamericano 21:75-90.

Holdaway, S.; J. Shiner y P. Fanning. 2004. "Hunter-gatherers and the archaeology of discard behavior: an analysis of surface stone artifacts from Sturt National Park, Western New South Wales, Australia”. Asian Perspectives 43(1):34-72.

Manzi, L.; C. Favier Dubois y F. Borella. 2009. "Identificación de agentes perturbadores y estrategias tendientes a la conservación del patrimonio arqueológico en la costa del Golfo de San Matías, provincia de Río Negro”. Intersecciones en Antropología 10:3-16.

Martínez, H.; C. Náñez, A. Lizuain, C. Dal Molín y A. Turel. 2001. "Hoja Geológica 4166-II, San Antonio Oeste. Provincia de Río Negro. Buenos Aires, Instituto de Geología y Recursos Minerales, Servicio Geológico Minero Argentino". Boletín 254: 32. 
Oresanz, J.; A. Escofet y V. Scarabino. 1973. "Relevamiento y tipificación de las comunidades bentónicas". En Relevamiento ecológico y tipificación de las comunidades del litoral maritimo de la provincia de Río Negro, con especial referencia al establecimiento de áreas de cultivo para especies de interés comercial, pp. 295-362. Instituto de Biología Marina - Asesoría de Desarrollo de Río Negro - Consejo Federal de Inversiones, Buenos Aires.

R Development Core Team. 2005. R: A Language and Environment for Statistical Computing, Reference Index Version 2.2.1. R Foundation for Statistical Computing, Vienna.

Sánchez, L. 1973. "Geología litoral”. En Relevamiento ecológico y tipificación de las comunidades del litoral maritimo de la provincia de Río Negro, con especial referencia al establecimiento de áreas de cultivo para especies de interés comercial, pp: 112-141. Instituto de Biología Marina - Asesoría de desarrollo de Río Negro - Consejo Federal de Inversiones, Buenos Aires.

Shelley, P. 1993. "A geoarchaeological approach to the analysis of secondary lithic deposits". Geoarchaeology: An International Journal 8(1): 59-72.

Shiner, J. 2004. Place as occupational histories: towards an understanding of deflated surface artefact distributions in the West Darling, New South Wales, Australia. Tesis para optar al grado de Doctor of Philosophy in Anthropology, Department of Anthropology, University of Auckland.

Shiner, J.; S. Holdaway, H. Allen y P. Fanning. 2005. "Stone artifact assemblage variability in late Holocene contexts in western New South Wales: Burkes Cave, Stud Creek and Fowlers Gap". En Lithics 'Down Under': Australian perspectives on lithic reduction, use and classification, editado por C. Clarkson y L. Lamb, pp: 67-80. British Archaeological Reports International Monograph Series. Archaeopress, Oxford.

Symons, J. 2003. "Obsidian artefacts and land-use in the mid-Holocene of the Willaumez Peninsula, Papua New Guinea”. Australian Archaeology 57:128134. 